'Programa de Estudios Médicos Humanísticos,

Facultad de Medicina,

Pontificia Universidad Católica de Chile.

${ }^{2}$ Departamento de

Salud Pública, Escuela

de Medicina, Facultad

de Medicina, Pontificia

Universidad Católica de Chile.

aDoctor en Filosofía y

Doctor en Bioética.

${ }^{b}$ Doctor en Bioestadísticas.

Fuentes de financiamiento:

Facultad de Medicina y

Facultad de Educación,

Pontificia Universidad

Católica de Chile, en forma

de apoyo financiero. Esas

Facultades no tuvieron

influencia en: el diseño del

estudio; la recolección, el

análisis o interpretación de

los datos; la preparación,

revisión o aprobación del manuscrito.

Recibido el 30 de mayo de

2011, aceptado el 27 de octubre de 2011.

Correspondencia a:

Dr. Francisco Mardones

Departamento de Salud

Pública. Pontificia Universidad

Católica de Chile.

Marcoleta 434,

Santiago, Chile.

Teléfono: 56 (2) 3546898,

Fax: 56 (2) 6331840.

mardones@med.puc.cl

\section{Relación médico-paciente en la Pontificia Universidad Católica de Chile: evaluación de una escala de medición}

\author{
ÁNGEL RODRÍGUEZ ${ }^{1, a}$, FRANCISCO MARDONES ${ }^{2}$, LUIS VILLARROEL ${ }^{2, b}$
Evaluation of a scale to assess physician-patient relations

Background: Beneficence, respecting autonomy of patients to make their own decisions, is crucial for good physician-patient relations (PPR), a leading objective in health care. Aim: To validate a previously designed scale to assess PPR in Chile. Material and Methods: A scale with 55 questions grouped in six dimensions, was applied to a convenience sample of 146 individuals, composed by physicians, patients and medical students, at the school of medicine from the Pontificia Universidad Católica de Chile (PUC). Internal consistency (Alpha of Cronbach) of answers was analyzed. The existence of correlations between answers that may justify the application of a factorial analysis was assessed using Bartlett and Kaiser-Meyer-Olkin (KMO) tests. Factorial analysis was used to identify specific dimensions and reduce the number of questions. Results: Factorial analysis performed in 125 subjects with complete answers allowed to reduce the scale to 28 questions, grouped in six dimensions. Cronbach alpha value was 0.78. Bartlett test was highly significant $(p<0.0001)$, and KMO score was 0.784, considered as meritorious. Conclusions: The validated scale will allow the performance of new studies among physicians and patients, to assess and compare their respective scores.

(Rev Med Chile 2012; 140: 176-185).

Key words: Personal autonomy; Physician-patient relations; Questionnaires.
E ste artículo se refiere a la relación médicopaciente (RMP) frente a la posible deshumanización de la medicina. Se ha propuesto que el médico debe atender al paciente con beneficencia, respetando la autonomía de éste haciéndolo participar en la toma de decisiones ${ }^{1}$. Por ello, debe explicarle al paciente su diagnóstico y tratamiento, esperando su consentimiento informado. Este es el modelo llamado de alianza en la RMP que puede librar al médico de muchos problemas de carácter jurídico y económico, permitiendo que el paciente viva el proceso de su enfermedad con mayor serenidad y confianza.

La literatura nacional e internacional con respecto a estudios similares es escasa. Se encontró sólo un estudio de características parecidas en Chile, hecho en escala mucho menor, en médicos de hospitales públicos desde la perspectiva sicológica $^{2,3}$. En tres estudios realizados en Canadá el método fue la entrevista individual semiestructurada para ver cómo perciben los docentes de medicina la RMP y cómo se perciben ellos a sí mismos como modelos de aprendizaje de esta relación ${ }^{4-6}$. En Méjico, en el 2004 se hizo un estudio comparativo entre las prácticas y opiniones de médicos de Méjico y Estados Unidos de Norteamérica, sobre el consentimiento informado ${ }^{7}$. Más tarde en el 20052006, se realizó, también en Méjico, otro estudio con una muestra de 1.552 pacientes entrevistados después de salir de la consulta clínica y dirigido fundamentalmente a conocer el cumplimiento del consentimiento informado ${ }^{8}$.

La humanización de la salud necesita partir desde la cabecera del enfermo. En esa situación, se 
realizó durante el año 2006 la investigación sobre la visión de médicos, pacientes y alumnos de $6^{\circ} \mathrm{y}$ $7^{\circ}$ año de la escuela de medicina en cuanto la RMP, en campos clínicos de la Pontificia Universidad Católica de Chile (PUC). Los antecedentes de la investigación están presentados en un libro de reciente aparición sobre la tesis de doctorado que se condujo con parte de la información de la misma ${ }^{9}$. En este artículo se presenta la validación de la encuesta propuesta en la tesis para conocer las características y los modelos de la relación médico-paciente que podrían estar desarrollándose en Chile.

\section{Métodos}

En este estudio descriptivo-exploratorio se describen y analizan relaciones sobre la actual mirada de la RMP, del médico, del paciente y de los alumnos de $6^{\circ}$ y $7^{\circ}$ año de la Escuela de Medicina de la PUC ${ }^{10}$. A ellos se les aplicó la encuesta que se describe más abajo con el objetivo de validarla; esto fue realizado por cuatro médicos colaboradores. Antes de aplicarla se probó la comprensión y exactitud del lenguaje con un grupo de médicos y también a través de una aplicación piloto a pacientes.

El cuestionario fue aplicado en el hospital clínico PUC y en el hospital Sótero del Río a médicos y pacientes. Los alumnos estaban todos ubicados en el hospital clínico. La muestra en estudio fue de: 44 médicos, de los cuales 35 eran hombres y 9 mujeres, pertenecientes a 11 especialidades, con un rango de edad entre 50 y 60 años. Los estudiantes fueron 50 , de los cuales 29 eran de $6^{\circ}$ año y 21 de $7^{\circ}$ con un rango de edad entre 22 y 27 años; 24 eran hombres y 26 mujeres. Los pacientes fueron 52, de los cuales 24 eran hombres y 28 mujeres, $71 \%$ estaban hospitalizados y $28,8 \%$ eran pacientes ambulatorios; $50 \%$ tenía una edad igual o mayor de 60 años y $50 \%$ tenía una edad entre 30 y 50 años. En cuanto a la proveniencia de los 52 pacientes, $75 \%$ lo fueron del Hospital Clínico UC y 25\% eran del Sótero del Río.

Las especialidades médicas fueron las siguientes: 13 de Medicina Interna (29,5\%); 10 de Ginecología y Obstetricia (22,7\%); 7 de Salud Pública (15,9\%); 4 de Traumatología (9,15\%); 2 de Anatomía Patológica (4,5\%); 2 de Anestesiología $(4,5 \%) ; 2$ de Gastroenterología (4,5\%) 1 de Cardiología, Pediatría, Neurocirugía, respectivamente (2,3\% cada especialidad). Del total de la muestra $36(80 \%)$ eran del Hospital Clínico y 8 (20\%) del Hospital Sótero del Río.
La calidad de la RMP fue estimada a través de un cuestionario que agrupó 55 preguntas en 3 dimensiones ${ }^{1}$. Las respuestas a cada pregunta fueron valoradas con una escala tipo Likert de cinco grados que varió entre muy en desacuerdo, en desacuerdo, medianamente en acuerdo, en acuerdo y muy de acuerdo; tuvieron entre 1 y 5 puntos. Las preguntas se presentan en la Tabla 1 en cada una de sus dimensiones específicas.

Se pueden distinguir los aspectos conceptuales en la RMP de los aspectos vivenciales en la misma. Las dimensiones 1 y 2 se refieren a la evaluación de los conceptos o ideas que los médicos o pacientes tienen de la RMP mientras que la dimensión 3 expresa en cinco modelos de RMP la vivencia que ellos pueden tener de la misma ${ }^{10}$; la inclusión de los modelos en la dimensión 3 permitirá su comparación en nuevos estudios con el modelo de alianza y conocer su aceptación cuando se dan varias opciones. A continuación se explican las dimensiones iniciales del estudio:

1. Dimensión deshumanización de la salud: preguntas 1 a 13. Se definió como un conjunto de factores que han llevado a un reduccionismo de la medicina, empobreciendo la RMP, dejando fuera aspectos importantes inherentes a la condición humana ${ }^{10}$.

2. Dimensión antropológica: preguntas 14 a 22 . Se definió como la concepción del ser humano que se puede tener presente en la práctica médica, pudiendo ir desde una concepción trascendental-religiosa a una materialistaexistencialista ${ }^{10}$.

3. Dimensión Relación médico-paciente: preguntas 23 a 51. Se postularon para ser consultados cinco modelos de relación médico-paciente que aparecen como aquellos que concitan mayor acuerdo en la revisión del estado del arte y que permiten una caracterización más adecuada de la RMP ${ }^{10}$ :

3.1. Modelo paternalista o hipocrático: preguntas 23 a 28 . Hoy se considera que un médico tiene actitud paternalista cuando utiliza sus capacidades para determinar la condición del paciente y su estado en el proceso de la enfermedad identificando las pruebas y tratamiento que más probablemente restauren la salud del paciente o disminuyan el dolor; presenta la información seleccionada y exhorta al paciente a consentir en la intervención que él considera mejor ${ }^{11}$. 
Evaluación de una escala de medición sobre la relación médico-paciente - A. Rodríguez et al

\section{Tabla 1. Cuestionario para los tres actores. Contiene siete dimensiones y 55 preguntas}

\section{1) Dimensión deshumanización de la medicina:}

1. La alta eficacia de los avances científicos y técnicos en medicina, hacen innecesario tomar en cuenta la opinión y el sentir de los pacientes

2. Como los criterios de la ciencia y la fe religiosa se contradicen, el trabajo del médico debería centrarse solamente en la enfermedad del paciente

3. El médico es quien determina lo mejor para el paciente

4. El médico debe centrarse solamente en tratar al paciente en función de su enfermedad

5. Un documento legal firmado ante notario sobre deberes y derechos, mejoraría la relación entre el médico y el paciente

6. Es el paciente, como consumidor, quien tiene el derecho de tomar decisiones respecto de su enfermedad

7. La relación entre el médico y el paciente se basa en el voto de confianza que el paciente le da al médico

8. El médico debe informar de modo gradual, claro y veraz sobre la enfermedad al paciente, aunque ésta sea grave o fatal

9. El compromiso de confidencialidad implica que el médico guardará en secreto aspectos personales e íntimos que el paciente desea que se resguarden

10. El médico actúa como consejero, el paciente es el que toma la decisión según sus valores

11. La atención de salud debería seguir las leyes de la oferta y la demanda, como ocurre con otras actividades comerciales y servicios

12. El paciente puede demandar al médico, cuando cree que éste no cumple con su trabajo

13. En la relación entre el médico y el paciente, el médico sólo ve al paciente como un sujeto de estudio

\section{2) Dimensión antropológica:}

14. El enfermo terminal puede decidir cuándo acabar con su vida

15. La relación entre el médico y el paciente hoy tiene su principal eje en escuchar activamente al paciente por sobre los procedimientos técnicos

16. El paciente requiere ser tratado por el médico integralmente, considerando desde lo físico hasta lo espiritual

17. La salud es un estado de equilibrio integral de todas las dimensiones de la persona, incluyendo la religiosa o espiritual

18. El paciente prefiere que el médico tome las decisiones, porque él es el experto

19. En la práctica médica cotidiana, los pacientes reciben una atención digna sin discriminar por su (condición de): hombre o mujer

20. En la práctica médica cotidiana, los pacientes reciben una atención digna sin discriminar por su (condición de): Homosexual (gay, lesbiana) u otras tendencias sexuales

21. En la práctica médica cotidiana, los pacientes reciben una atención digna sin discriminar por su (condición de): Edad.

22. En la práctica médica cotidiana, los pacientes reciben una atención digna sin discriminar por su (condición de): Nivel Socio-económico

23. En la práctica médica cotidiana, los pacientes reciben una atención digna sin discriminar por su (condición de): Religión

24. Una atención de salud que se guía por las leyes de la oferta y la demanda, pone en riesgo la calidad humana de la relación entre el médico y el paciente

25. En el caso de enfermedades terminales, la familia del paciente requiere ser atendida, confortada y orientada por parte del médico

26. La fe religiosa es un aspecto que queda fuera de la relación entre el médico y el paciente

\section{3) Dimensión RMP en el modelo paternalista:}

27. El médico debe entregar al paciente la información necesaria sobre su enfermedad y tratamiento, incluyendo riesgos y beneficios posibles

28. El paciente ve al médico como un experto en tratar su enfermedad

29. Lo espiritual fortalece y compromete mucho más el profesionalismo del médico

30. Hoy la medicina está más centrada en generar ganancias como si fuera un negocio que en atender las necesidades del paciente

31. El médico debe considerar las consecuencias legales antes de atender a un paciente en una situación de urgencia

32. El paciente debe seguir estrictamente las instrucciones del médico 

4) Dimensión RMP en el modelo tecnológico:
33. La tecnología y los avances científicos aseguran el éxito del trabajo médico
34. La relación con el paciente, es difícil para el médico separar lo emocional de lo estrictamente profesional
35. El médico debe proporcionar los detalles de las posibles alternativas de tratamiento, pero es el paciente quien debe decidir
36. La comunicación en la relación entre el médico y el paciente debe caracterizarse por ser positiva y esperanzadora
37. El paciente que puede pagar recibe mejor atención de salud
38. La medicina es una ciencia, por eso lo religioso es innecesario

\section{5) Dimensión RMP en el modelo legal:}
39. El médico sabe más que el paciente sobre su enfermedad
40. La mayor información disponible sobre enfermedades y/o tratamientos médicos, favorece el respeto de los derechos del paciente y el cumplimiento de los deberes del médico
41. El médico tiene que saber escuchar activamente y acoger las inquietudes del paciente
42. El paciente debería asesorarse con un abogado cuando está insatisfecho con la atención del médico
43. El médico interroga al paciente únicamente para diagnosticar y proponer un tratamiento
44. La relación entre el médico y el paciente es similar a la relación entre un agente de ventas y un comprador o cliente

\section{6) Dimensión RMP en el modelo comercial:}

45. El médico requiere manejar adecuadamente la comunicación no verbal (gestos, silencio, entre otros) en su interacción con el paciente

46. Dios es el responsable de dar la vida al ser humano

47. El médico debe adquirir un seguro médico como protección ante posibles demandas

48. El conocimiento que el paciente tiene de sí mismo, le permite tomar buenas decisiones con su médico sobre el tratamiento de su enfermedad

49. El paciente tiene derecho a que se le explique su enfermedad y tratamiento en un lenguaje adaptado a su condición sociocultural y anímica

50. Las expectativas del paciente deben ser consideradas en el proceso de diagnóstico y tratamiento por parte del médico.

\section{7) Dimensión RMP en el modelo de alianza:}

51. El médico tiene que manejar sus emociones, incluso en situaciones extremas, para comunicarse clara y objetivamente con el paciente

52. Es más importante en el trabajo médico, el tratamiento y diagnóstico de la enfermedad que poner atención a los problemas personales del paciente

53. El médico ve al paciente como alguien que necesita ayuda

54. En la consulta, el médico pone más interés en los exámenes que en escuchar activamente la vivencia de la enfermedad del paciente

55. El paciente ve a su médico, por su profesión y lo que sabe, como una autoridad

3.2. Modelo tecnológico: preguntas 29 a 34. En este modelo se acentúa el carácter "científico" del médico quien se interesa en el paciente en cuanto a un "caso", ignorando completamente los aspectos humanos de la relación con el enfermo ${ }^{11}$. 3.3. Modelo legal: preguntas 35 a 41. Este modelo se caracteriza, porque el médico y el paciente firman un contrato legal de común acuerdo, que limita la naturaleza de la relación ${ }^{11}$.

3.4. Modelo comercial o consumista: preguntas 41 a 46. En este modelo, el médico es un agente comercial, el paciente es un consumista de la medicina cuya autonomía en esta relación es absoluta, y la medicina, como consecuencia, queda sujeta a la economía de mercado ${ }^{12}$.

3.5. Modelo de alianza: preguntas 47 a 51. Este modelo es un paradigma tan antiguo como la tradición hipocrática. Indica una relación de colaboración y de acuerdo recíproco sobre cuestiones que respectan a la cura de la salud. La alianza médico-paciente indica un acuerdo sobre aspectos de la medicina que son todo menos que extrínsecos respecto a las dimensiones humanas. Los aspectos legales o contractuales se exigen por el hecho de que la relación médico-paciente está dentro de un contexto de relaciones públicas, 
pero que no constituyen la base de aquellas cosas que vienen antes y que se retienen más importantes ${ }^{13}$. Para que un modelo de relación médico-paciente se acerque más a una relación de equilibrio entre la confianza que el paciente otorga al médico tratante y su capacidad de tomar decisiones tras haber sido informado, ha de proveer las bases para que se establezca un diálogo entre el médico y el paciente y una relación de mutua confianza, desinteresada. En circunstancias ordinarias, el tomar decisiones es tarea de ambos, médico y paciente, respetando siempre la integridad de la persona. El médico toma decisiones por y con el paciente, no en lugar del paciente ${ }^{14}$.

Las sub-muestras de médicos, pacientes y estudiantes, fueron de conveniencia y no son representativas de algún universo. Ellas fueron consideradas como un solo grupo dado que la encuesta se pretende que sea útil para detectar la calidad de la RMP en cualquiera de los tres grupos incluidos. Se utilizó el coeficiente alfa de Cronbach para determinar la consistencia interna del instrumento ${ }^{15}$. Se utilizó la prueba de esfericidad de Bartlett y el valor de Kaiser-Meyer-Olkin (KMO) para determinar la existencia de correlación entre las variables que justificara la realización de un análisis factorial ${ }^{16}$. Se realizó un análisis factorial por método de componentes principales con rotación Varimax para identificar las dimensiones en el instrumento y para reducir el número de preguntas ${ }^{17}$.

Este estudio fue aprobado por el Comité de ética de la Escuela de Medicina de la Pontificia Universidad Católica de Chile. Los archivos fueron confidenciales y los participantes sólo fueron identificados con un código.

\section{Resultados}

De los 146 sujetos reclutados, 21 no respondieron alguna de las 56 preguntas. Se realizó el análisis factorial con 125 sujetos.

El coeficiente alfa de Cronbach para las respuestas a las 55 preguntas tuvo un valor de 0,847 , indicando que $84,7 \%$ de la variabilidad de las respuestas obtenidas entre 1 y 5 puntos representa diferencias verdaderas entre las personas y $15,3 \%$ refleja fluctuaciones al azar. Los resultados indicaron que las preguntas son homogéneas y que el instrumento tiene consistencia interna ya que no se modificó sustancialmente el puntaje del alfa de Cronbach si se elimina alguna de las mismas.

En el análisis factorial, la prueba de Bartlett tuvo un valor $\mathrm{p}<0,0001$, negando la esfericidad en la matriz de preguntas o elementos. Es decir, existe correlación entre algunas preguntas, lo que permite identificar dimensiones que las agrupen justificando la realización de un análisis factorial.

El análisis factorial permitió reducir a 28 preguntas el instrumento e identificar con el método de Varimax siete dimensiones que explican en su conjunto $64,4 \%$ de la varianza de los datos. Los valores de las correlaciones de cada pregunta seleccionada con las 7 dimensiones identificadas fueron todas iguales o mayores de 0,493 (Tabla 2). En el caso de la dimensión 7 se identificó sólo la pregunta 30 con un valor de $\mathrm{r}$ mayor de 0,493. Esa pregunta se asignó a la dimensión 1, dado que se requieren al menos tres preguntas con alta correlación para configurar una dimensión; además, en la dimensión 1 la pregunta 30 tiene un valor de $r$ discretamente inferior al observado en la dimensión 7. Por lo tanto, quedaron sólo seis dimensiones en la encuesta. Los nombres de cada una de las seis dimensiones según la nueva agrupación de preguntas se presentan también en la Tabla 3 con la identificación de las anteriores.

El análisis de fiabilidad final con las 28 preguntas tuvo un alfa de Cronbach de 0,775 algo menor que lo obtenido para las 55 preguntas. Las preguntas son homogéneas y el instrumento tiene consistencia interna ya que no se modificó sustancialmente el puntaje del alfa de Cronbach aun si se elimina cualquiera de las 28 de las mismas; el valor final de 0,775 solamente se mueve entre uno y tres centésimos de punto en algunas de las preguntas mientras que en cuatro casos se mantiene. El nuevo análisis factorial con 28 preguntas mostró que la prueba de Bartlett tuvo un valor $\mathrm{p}<0,0001$, negando también la esfericidad en la matriz de preguntas o elementos. La medida de adecuación muestral de Kaiser-Meyer-Olkin (KMO) llegó a 0,784, indicando que las variables consideradas en el estudio miden factores comunes, lo cual también es indicador de la adecuación del análisis factorial. Un valor de la prueba KMO cercano a 0.8 se considera meritorio ${ }^{18}$.

Dado que la Escala de Likert utilizada tiene 5 opciones fue necesario indagar si al tener un número impar de alternativas de respuesta pudo 
ARTÍCULOS DE INVESTIGACIÓN

Evaluación de una escala de medición sobre la relación médico-paciente - A. Rodríguez et al

Tabla 2. Correlación de las 28 preguntas seleccionadas y cada una de las 7 dimensiones identificadas; se eliminó luego la dimensión 7 sobre el modelo comercial (en negrilla se destacan las preguntas seleccionadas para cada dimensión)

\begin{tabular}{|c|c|c|c|c|c|c|c|}
\hline Pregunta & $\begin{array}{c}\text { Dim1 } \\
\text { Modelo } \\
\text { paternal. }\end{array}$ & $\begin{array}{c}\text { Dim2 } \\
\text { Concepc. } \\
\text { Antrop. }\end{array}$ & $\begin{array}{c}\text { Dim3 } \\
\text { Deshum. } \\
\text { de la } \\
\text { medicina }\end{array}$ & $\begin{array}{c}\text { Dim4 } \\
\text { Modelo } \\
\text { legal }\end{array}$ & $\begin{array}{c}\text { Dim5 } \\
\text { Modelo } \\
\text { alianza }\end{array}$ & $\begin{array}{c}\text { Dim6 } \\
\text { Modelo } \\
\text { autonom. }\end{array}$ & $\begin{array}{c}\text { Dim7 } \\
\text { Modelo } \\
\text { comerc. }\end{array}$ \\
\hline p. 1 & 0,680 & $-0,024$ & 0,005 & 0,025 & 0,177 & 0,036 & 0,019 \\
\hline p. 2 & 0,665 & 0,224 & 0,007 & 0,237 & 0,164 & 0,016 & 0,334 \\
\hline p. 3 & 0,766 & 0,100 & 0,073 & 0,168 & $-0,044$ & 0,173 & 0,268 \\
\hline p. 4 & 0,688 & 0,241 & 0,003 & 0,328 & 0,162 & 0,039 & 0,190 \\
\hline p. 5 & 0,307 & $-0,007$ & $-0,135$ & 0,619 & 0,181 & 0,282 & $-0,046$ \\
\hline p. 7 & $-0,019$ & 0,157 & $-0,176$ & $-0,213$ & 0,682 & $-0,034$ & 0,176 \\
\hline p. 8 & 0,114 & 0,042 & 0,077 & $-0,052$ & 0,861 & 0,049 & $-0,023$ \\
\hline p. 9 & 0,118 & $-0,017$ & 0,157 & 0,146 & 0,724 & 0,276 & $-0,150$ \\
\hline p. 10 & $-0,153$ & $-0,015$ & $-0,263$ & $-0,190$ & $-0,111$ & $-0,691$ & 0,035 \\
\hline p. 16 & 0,113 & 0,826 & $-0,091$ & 0,213 & 0,124 & $-0,070$ & $-0,136$ \\
\hline p. 17 & 0,108 & 0,810 & $-0,079$ & 0,138 & $-0,029$ & $-0,008$ & $-0,148$ \\
\hline p. 18 & 0,735 & 0,078 & $-0,087$ & 0,035 & $-0,059$ & 0,064 & $-0,136$ \\
\hline p. 19 & $-0,023$ & 0,002 & 0,625 & $-0,035$ & $-0,004$ & $-0,117$ & $-0,493$ \\
\hline p. 20 & 0,056 & $-0,050$ & 0,819 & 0,016 & 0,056 & 0,098 & 0,106 \\
\hline p. 21 & $-0,011$ & $-0,119$ & 0,848 & $-0,122$ & 0,027 & 0,049 & 0,016 \\
\hline p. 22 & 0,101 & $-0,065$ & 0,794 & $-0,097$ & 0,010 & 0,093 & 0,196 \\
\hline p. 23 & $-0,105$ & 0,065 & 0,766 & $-0,093$ & $-0,040$ & 0,044 & $-0,171$ \\
\hline p. 25 & 0,015 & 0,736 & $-0,086$ & $-0,058$ & 0,144 & 0,253 & 0,066 \\
\hline p. 29 & 0,123 & 0,704 & 0,057 & $-0,033$ & $-0,022$ & 0,227 & 0,218 \\
\hline p. 33 & 0,496 & 0,111 & 0,110 & 0,199 & 0,010 & 0,030 & 0,560 \\
\hline p. 38 & 0,212 & 0,600 & 0,012 & 0,155 & 0,022 & $-0,075$ & 0,375 \\
\hline p. 39 & $-0,622$ & 0,048 & $-0,097$ & 0,273 & 0,222 & $-0,310$ & 0,110 \\
\hline p. 42 & 0,035 & 0,034 & $-0,195$ & 0,821 & $-0,102$ & 0,009 & 0,039 \\
\hline p. 43 & 0,282 & 0,172 & $-0,028$ & 0,525 & $-0,081$ & 0,066 & 0,060 \\
\hline p. 44 & 0,031 & 0,348 & 0,006 & 0,493 & $-0,141$ & $-0,066$ & 0,324 \\
\hline p. 48 & 0,083 & 0,133 & 0,002 & 0,010 & 0,106 & 0,808 & 0,000 \\
\hline p. 50 & 0,200 & 0,480 & 0,002 & 0,025 & 0,012 & 0,545 & 0,222 \\
\hline p. 52 & 0,605 & 0,347 & $-0,015$ & 0,255 & 0,152 & 0,024 & $-0,003$ \\
\hline
\end{tabular}

ocurrir que los sujetos respondan con una tendencia mayor en la respuesta del medio o central, en este caso el número 3. Esta posibilidad fue descartada dado que de las 28 preguntas seleccionadas solamente en 4 de ellas la opción 3 fue la preferida.

\section{Discusión}

Este estudio permitió validar el nuevo instrumento, reduciendo el número de preguntas y dimensiones, obteniéndose una fiabilidad alta del mismo. El alto valor del índice KMO indicó que las preguntas de este instrumento están midiendo factores comunes y que se pueden agrupar, lo que se hizo con aquellas dimensiones donde las preguntas tuvieron una alta correlación.

Los dos análisis anteriores realizados en Chile se refirieron a la exploración cualitativa de la satisfacción profesional en dos muestras de médicos y no construyeron un instrumento de evaluación de la RMP ${ }^{2,3}$. Tampoco concluyeron en una encuesta validada los estudios previos de la RMP realizados en otros países, lo que impide su comparación con el presente estudio ${ }^{4-8}$. Los aspectos incluidos en esos estudios fueron referidos más bien a la autonomía del paciente y no consideraron aspectos más globa- 
Evaluación de una escala de medición sobre la relación médico-paciente - A. Rodríguez et al

Tabla 3. Cuestionario para los tres actores. Contiene seis dimensiones y 28 preguntas.

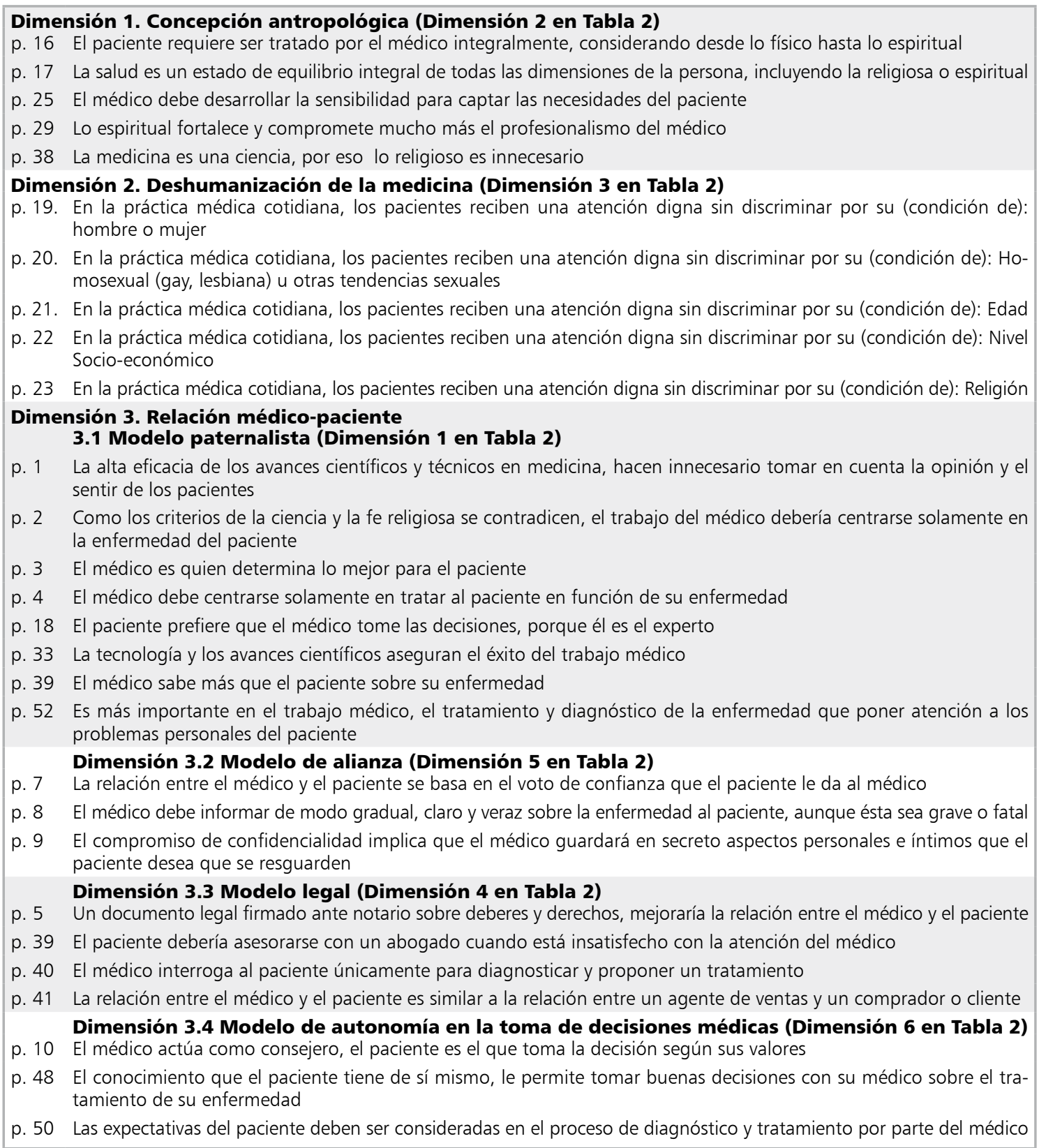

les como los de este nuevo instrumento que se interesa más bien en la humanización de la medicina.

En los modelos de RMP seleccionados y validados en este estudio hay dos que hacen referencia al principio de autonomía del paciente (modelo legal y modelo de autonomía en la toma de decisiones médicas) y dos que hacen referencia más bien al principio de beneficencia (modelo paternalista y modelo de alianza). En este estudio se ha seguido utilizando el concepto de "alianza", tal como lo han hecho los especialistas en bioética de la Universidad de Georgetown ${ }^{19,20}$. Debe notarse que las 
preguntas consideradas en la propuesta inicial como parte de las dimensiones conceptuales o de las vivenciales no se comportaron de esa manera en la propuesta final sino que se entremezclaron completamente. Ello indica que la separación inicial no era adecuada y que la validación cumplió el propósito de ubicar las preguntas en sus dimensiones más precisas, tanto conceptuales como vivenciales.

Dentro de las limitaciones de este estudio, destaca la mayor representación de pacientes hospitalizados (71\%), lo cual podría hacer diferencia en la RMP, si se compara con la medicina ambulatoria. Sin embargo, nuevos estudios pueden resolver esta dificultad y otras, como se explica en el siguiente párrafo. En la sección Resultados se descartó otra posible limitación relativa a una posible concentración de respuestas en el nivel medio de la escala de Likert.

Dado que no existía una encuesta de este tipo en nuestro medio, este instrumento validado queda ahora útil para realizar nuevos estudios representativos de poblaciones de médicos y/o pacientes en los sectores públicos y privados tanto hospitalizados como no hospitalizados, para empezar a comparar los puntajes respectivos al interior o al exterior de las instituciones. La reflexión sobre los nuevos estudios seguramente permitirá humanizar la RMP en los aspectos que se detecten más débiles. Es notorio que las dimensiones seleccionadas expresan aspectos conceptuales y vivenciales diversos que deben especificarse en las comparaciones respectivas. No se pueden hacer comparaciones del total del puntaje.

\section{Referencias}

1. Rodríguez A. Relación médico-paciente en algunos campos clínicos de la Pontificia Universidad Católica de Chile. Santiago de Chile: Andros Impresores; 2010. p. 222.

2. Horwitz N. El cambio en la práctica médica. Desafíos psicosociales para la profesión. Rev Med Chile 2004; 132: 768-72.

3. Bascuñán ML. Cambios en la relación médico-paciente y nivel de satisfacción de los médicos. Rev Med Chile 2005; 133: 11-6.

4. Côtè L, Leclère H. How clinical teachers perceive the doctor-patient relationship and themselves as role models. Acad Med 2000; 75: 1117-24.

5. Maheux B, Beaudoin C, Berkson L, Côtè L, Des Marchais
J, Pierre J. Medical faculty as humanistic physicians and teachers: the perceptions of students at innovative and traditional medical schools. Med Educ 2000; 34: 630-4.

6. Beaudoin C, Maheux B, Côté L, Des Marchais JE, Jean $\mathrm{P}$, Berkson L. Clinical teachers as humanistic caregivers and educators: perceptions of senior clerks and secondyear residents. CMAJ 1998; 159: 765-9.

7. Hall RT, Rosencrance G, Sypher B. La relación médicopaciente en Méjico y en Los Estados Unidos: un estudio empírico. Perspectivas Bioéticas 2004; 9: 103-26.

8. Hall RT, Hernández JL, Carranza O, Serrano FJ, Martínez JA, Rosencrance G, et al. Relaciones médico-paciente: un estudio empírico. Medicina y ética 2007; 18: 315-36.

9. Rodríguez A, Relación médico-paciente en algunos campos clínicos de la Pontificia Universidad Católica de Chile. ED. Santiago de Chile: Andros Impresores; 2010. p. 122-31.

10. Rodríguez Guerro, A. Relación médico-paciente en algunos campos clínicos de la Pontificia Universidad Católica de Chile. Roma: Ed. Morolo, Ateneo Pontificio Regina Apostolorum - IF press; 2009, p. 164-215.

11. Rodríguez Guerro A. Modelos de la relación médicopaciente. Reflejo de la deshumanización de la salud. Ars Medica (Facultad de Medicina, Pontificia Universidad Católica de Chile) 2006; 12: 101-3.

12. Emanuel EJ, Emanuel LL. Four Models of the PhysicianPatient Relationship. JAMA 1992; 267: 2221-6.

13. Russo G. Il medico. Identità e ruoli nella società di oggi. Roma: CIC Edizione Internazionali; 2004. p. 16971.

14. Rodríguez Yunta E. Relación médico-paciente y práctica médica. En: Diálogo y cooperación en salud, diez años de bioética en la OPS. Fernando Lolas Stepke, ed. Serie Publicaciones Unidad de Bioética IKM OPS/OMS. Santiago de Chile: Andros Impresores; 2004, p. 61-73.

15. Everitt B, Howell D. Encyclopedia of Statistics in Behavioral Science (Vol.2) Chichester: John Wiley \& sons, Ltd.; 2005.

16. Hinton P. Statistics Explained - A Guide for Social Science Students (2nd Ed). London: Psychology Press; 2004.

17. Stevens J. Applied multivariate statistics for the social sciences (4th Ed). Mahwah, New Jersey: Lawrence Erlbaum Associates, Publishers; 2002.

18. Kaiser HF. An index of factorial simplicity. Psychometrika 1974; 31: 31-6.

19. Beauchamp TL, Childress JF. Paternalism: conflict between beneficence and autonomy. En: Principles of Biomedical Ethics. $5^{\text {th }}$ Ed. New York: Oxford University Press, 2001; 176-91.

20. Pellegrino ED, Thomasma DC. For the patient's good. The restoration of beneficence in health care. New York: Oxford University Press, 1988. 